\title{
A Modeling system on the Implementation of Automated Vehicles
}

\author{
Aaron Don M. Africa, Samuel Alexander Pasia, Jereme Adriane Sy \\ Department of Electronics and Communications Engineering \\ De La Salle University, Manila \\ 2401 Taft Ave., Malate, Manila 1004, \\ Philippines, aaron.africa@dlsu.edu.ph
}

\begin{abstract}
As time pass-by, technology improves and innovates with it. The newly introduced autonomous vehicles have their capability to do different actions where no human intervention is needed, such as automatic parking and crash collision avoidance. This study is all about autonomous vehicles (AV) that are capable of traveling and operating in the Philippines. The researchers aimed to determine whether an AV is apt to work in the country, given the hindrances such as traffic and various driving and road conditions. To do that, they considered past studies that relate and may help in their research, such as the use of heading reference assisted pose and controllers. In analyzing the data, they made block models of their study that considers the given circumstances such as traffic and road - for instance, a block diagram if the $\mathrm{AV}$ is in an undeveloped area and if the AV is at an intersection. Following the data gathered and considering the block diagrams, the students conclude that the Philippines is not yet ready for autonomous vehicles. Lastly, the researchers give suggestions that may be keys for AV to work in the Philippines.
\end{abstract}

Key words: autonomous vehicles, traffic, road conditions, vehicle motion.

\section{INTRODUCTION}

Traffic is one of the most difficult problems to solve in the Philippines. In the past few years, there was an increase in the usage of motor vehicles and automobiles. For instance, from the year 2000 to 2009, there has been an increase of 3.2 million motorcycles that have been registered, which totals to over $320 \%$ of registered motor vehicles over 10 years. The further increase of vehicles every year will produce worse traffic than the Philippines is facing today [1].

In research by Montgomery (2018), a self-driving car or autonomous vehicle (AV) is a type of vehicle where it can drive and travel on its own. It uses different sensors for it to move from one point to another without human intervention. Through the years, there have been improvements in autonomous vehicles such as automatic parking, lane-following, lane-changing, and crash avoidance. In addition to these improvements, the autonomous vehicles itself has a benefit for the driver. For the reason that it can safely drive on its own, a driver can be free from the wheel or free from the control of the vehicle, which can enable the driver to do other tasks while the car is traveling. An autonomous vehicle can also be a key to the reduction of traffic congestion in a place. This type of vehicle can communicate with another autonomous car, therefore an incoming autonomous vehicle can reroute if an AV is stuck in the road it was supposed to take. Likewise, the AV can also communicate in traffic lights via a network, which can delay the time for an AV to move once the green light shows [2].

This research aims to determine whether an autonomous vehicle can be used in the Philippines, given the conditions of road and traffic. Furthermore, if this type of vehicle is feasible in the Philippines, the researchers will determine what can be innovated and improved for an autonomous vehicle to fully adjust the settings for the country.

\section{BACKGROUND OF THE STUDY}

Traffic congestion in the Philippines worsens every day since there are more residents buying cars instead of using public transport. With this increasing volume of vehicles every day, the major leading roads thus produce a higher percentage of traffic congestion [3]. Table 1 shows the travel speeds in different roads in Metro Manila, and their respective average speed through the years. This travel speed table was taken during daytime off-peak periods, 7 days per week, and for a year.

Table 1: Average travel speeds in major roads in the Philippines [1]

\begin{tabular}{|l|c|c|c|c|}
\hline \multirow{2}{*}{\multicolumn{1}{|c|}{ Road }} & \multicolumn{4}{c|}{ Travel speed (kph) } \\
\cline { 2 - 5 } & $\mathbf{2 0 0 8}$ & $\mathbf{2 0 0 9}$ & $\mathbf{2 0 1 0}$ & $\mathbf{2 0 1 1}$ \\
\hline Edsa & 32.78 & 36.24 & 32.07 & 36.09 \\
\hline Marcos Highway & 28.19 & 28.02 & 27.92 & 27.1 \\
\hline Road 10 & 28.97 & 32.54 & 36.78 & 36.67 \\
\hline Quezon Avenue & 27.73 & 26.36 & 32.58 & 32.34 \\
\hline McArthur Highway & No data & 19.99 & 17.31 & 18.21 \\
\hline $\begin{array}{l}\text { Commonwealth } \\
\text { Avenue }\end{array}$ & 35.93 & 36.06 & 41.54 & 39.35 \\
\hline C5 & No data & 27.84 & 35.24 & 29.37 \\
\hline
\end{tabular}

Normally, an autonomous vehicle can be designed with embedded control systems. In a paper by [4], they stated that a self-driving car can be designed just like a robot, through an 
embedded control system and behavior-based robot. Designing control systems for field robots, receptors, effectors, communication buffers and a control subsystem works hand in hand [5]. These compositions must work well because these will lead to an increase in reliability and working conditions. Figure 1 represents how a robotic system works internally. This shows how a control subsystem, receptors (both virtual and real), and effector (both virtual and real) performs. Figure 2 presents how a control subsystem behaves with another subsystem, in which they coordinate with each other. Lastly, Figure 3 displays a state diagram on how behavior-based automation works.

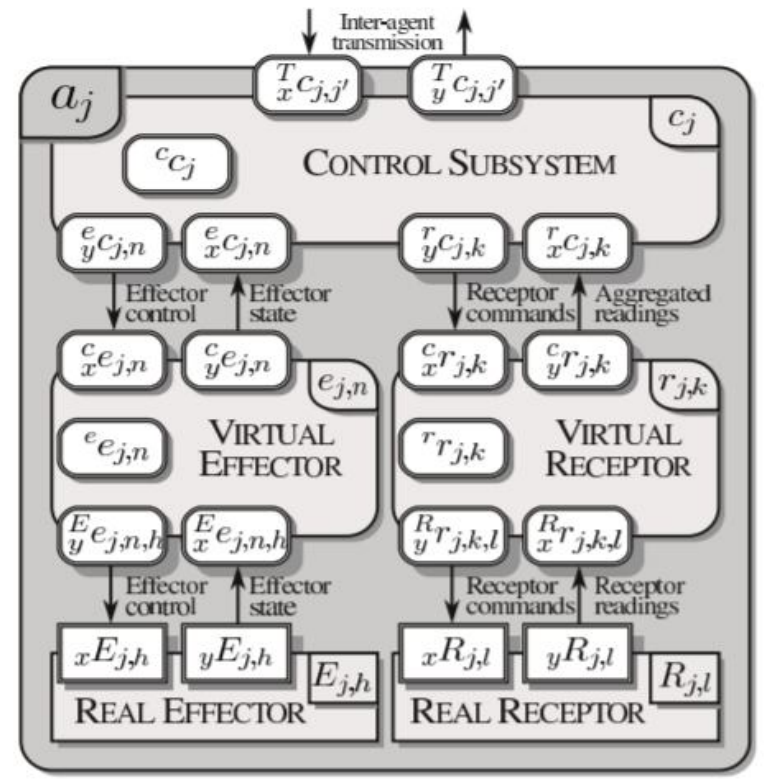

Figure 1: Internal performance of a robotic system [4]

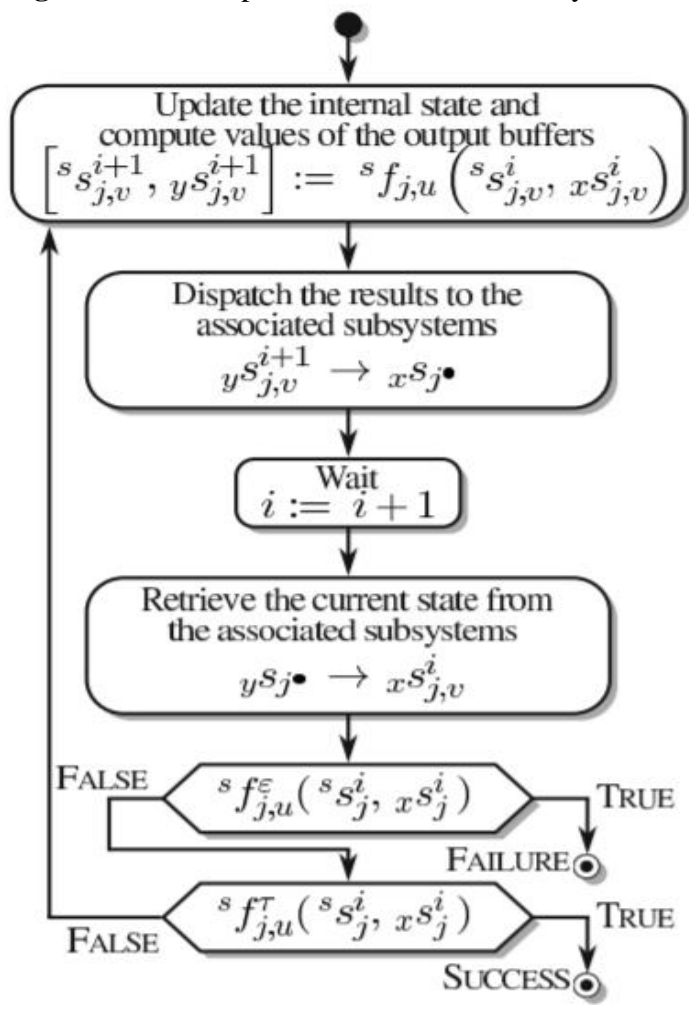

Figure 2: Flowchart of a control subsystem coordinating with another control subsystem [4]

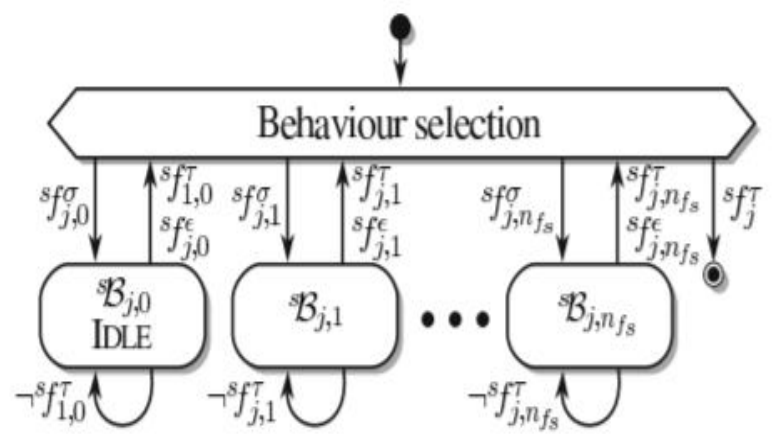

Figure 3: State diagram of a behavior-based robotic system [4]

Robot programming can be an important role in the study [6]. Robot programming by demonstration or $\mathrm{PbD}$ can be divided into three parts. Imitation learning, training machine, and modeling of perception and action. The paper of [7] that programming and kinematics application are difficult tasks to do, that is why a control scheme for assisting this kinetics are important. In the research by [8], PbD can be a set of rules and demonstrations that the user can program, and the machine can learn from its previous actions. These successions can be useful for the AV to learn and innovate as it moves in the various road conditions. With these researches, robot programming and control systems applications can be used for further implementation of the project [8]. $\mathrm{PbD}$ is also useful in research by [9] since the learning of robots and machines can be implemented for future use.

With the topics introduced, the researchers would like to determine whether self-driving cars are applicable in a country like the Philippines. To achieve this, they will design a control system that is fully capable to work in a road condition where traffic is evident. Conditions such as traffic, road conditions and will be considered since this paper will tackle on the feasibility of autonomous vehicles in the Philippines.

\section{STATEMENT OF THE PROBLEM}

The idea of the autonomous vehicle or the self-driving car is still being developed today. Although there are no fully automated and commercially available vehicles, there are already vehicles that are partially automated which means that there are some functions that the vehicle can do without requiring human assistance. With this, the students want to determine if autonomous vehicles can be implemented in the Philippines given its difficult driving conditions. According to [10], the Philippines has been ranked the worst city to drive in given that it takes a motorist 4.9 minutes to drive a kilometer while being compared to other countries such as Colombia and Indonesia which only takes less than 4 minutes. The traffic is mainly due to poor roads and road network, urban planning problems, and accidents [11]. These conditions might provide difficulty in the algorithms of the automation in vehicles which could also tell if automation can be used in the Philippines. 


\section{SIGNIFICANCE OF THE STUDY}

The Philippines is a country where a majority of its residents own vehicles. Statistically, there are 12,000 Filipinos that died because of major road accidents. These road accidents are commonly caused by over speeding, improper driving skills, bad overtaking, and driving under the influence of alcohol. Human drivers, on how they interact and how they manage whenever they are driving [12]. In research by [13], state of mind is important when it comes to driving. The research by [14] developed an analysis of the steering functions of a driver. These researches greatly deal in the study since they can also determine the driver's driving conditions. These common causes can be reduced and compensated since an autonomous vehicle has embedded control systems that cover all the causes. An autonomous vehicle has a control system in itself that calculates the speed when traveling to promote safety. The AV also has sensors that can sense whether it is safe to change lanes. For the reason that it is a self-driving car, the driver's input is no longer needed. Furthermore, the Philippines has a bad traffic condition, which can be reduced because of the abilities of an autonomous vehicle to communicate with another autonomous vehicle and traffic lights. Autonomous vehicles can also widen the perception and perspective of the driver [15].

Given the said statements, this study aims to determine whether autonomous vehicles are practically possible to use in the country. Additionally, this study can help different auto-manufacturing companies to adjust their designs for countries that have a severe traffic conditions, like the Philippines. This study can also benefit in reduction of traffic congestion in different places, since an autonomous car can communicate with another autonomous car and traffic light signals.

\section{DESCRIPTION OF THE SYSTEM}

For this paper, the students determine the working conditions of an autonomous vehicle by gathering sources and related literatures that are related to the research. Furthermore, the students designed block models for systems that are adaptable and feasible to the given circumstances such as traffic and road conditions. Additional explanations of the designed system are discussed in Methodology, Data and Results and Analysis.

\section{METHODOLOGY}

The students first gathered sufficient data from different studies that focus on the improvement of automated vehicles. The studies that they specifically looked for contains different algorithms to compensate for the difficulties the automated vehicle will encounter such as collisions and obstacle avoidance. With those algorithms, they have constructed a block diagram that will show how the automated vehicle will operate in the Philippines. Figure 4 shows the block diagram of the methodology.

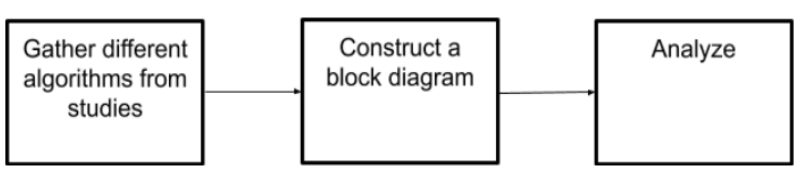

Figure 4: Block Diagram of the Methodology

\section{REVIEW OF THE RELATED LITERATURE}

The work of [16] focused on creating a control to prevent collisions of automated vehicles at road intersections. This was done by creating a supervisor algorithm in which the supervisor will activate when a future collision is to occur. The supervisor should be connected to a network of automated drivers for it to override them when a collision has been predicted.

As per [17], they used a different method for collision avoidance. Spline parameterization was applied to compute for the non-collision trajectories of the environment and can be used in linear and non-linear models.

Since the Philippines has poor urban planning in where buildings and structures can be built anywhere, automated vehicles may have a difficult time maneuvering in this type of environment. In the paper of [18], they made a Heading reference assisted pose estimation or (HRPE) where it used to compensate for the estimation error of the automation of vehicles when turning in an environment with poor features. This is done by setting an orientation as the heading reference and taking measurements from this reference from the sensors.

For vehicles that have driver-assist the research of [19], wanted to determine the probability of collision when a preceding vehicle is approaching a stop sign, a speed $\mathrm{p}$, or an intersection. It is because in these scenarios rear-end collisions are most likely to occur. Their team did this by doing simulations for every scenario and even considered the reaction time of the driver. They then found out that the probability of safety is within 5\% of the theoretical value.

As per [20], their study focused on autonomous miniature race cars. They used the viability kernel method so that the autonomous race cars can stay on track more efficiently. It was later found out that there is a control that keeps the system within the kernel. They concluded that this method provides a safer driving style but with a short increase in travel time.

When it comes to gear shifting in automated cars the research of [21], focused on the optimal gear shift schedule. This was made so that the vehicle minimizes fuel consumption while reaching the maximum amount of torque in shifting. They successfully did this by using a PI controller in regulating the motion of the vehicle and determines the required amount of torque.

For the work of [22], they wanted to develop a different MPC algorithm that is being used in today's automation in vehicles. They developed the Hamiltonian-switching hybrid nonlinear 
predictive control algorithm. They tested this algorithm on a racecar and the results show a better performance with the HaSH-NPC.

Advanced driving assist systems have been proven useful for drivers. It helps prevents accidents and guides the driver to a safer path. However, the main problem with these assisted systems is the authority of the control of the vehicle. The paper of [23] worked on the shared control of the driver and the lane keeping assist system. They did this in two parts, namely the tactical part and the operational part. The tactical part provides a smooth transition between the driver and the LKA system while the operational part is the one that computes the necessary actions in guiding the driver.

While driving on the road, there will inevitably be obstacles that the driver or the autonomous system will encounter. Because of this, the research of [24] created an algorithm to avoid obstacles for low speed autonomous vehicles. This was done using the barrier function method. When it predicts a collision will not occur then the barrier function is low. However, when it predicts that a collision is likely to occur then the program will take control making sure that the vehicle is guided safely.

Furthermore, in the work of [25], they used a controller which also uses the barrier function. The controller was done in two approaches which are an inner loop model and an outer loop model. The outer loop model generates a collision-free trajectory for the vehicle while the inner loop is a feedback controller that uses sensors as the input.

Vehicle localization is very important for AV's. It helps the autonomous driver to determine its location as well as the paths it needs to take. In the work of [26], they were able to create a localization scheme using camera-based lane recognition.

For [27], they wanted to consider the driver's action into the autonomous system. They first estimated for the steering wheel torque for the wheel and using the estimation, they trained driving machines with simulated and real driving data sets.

In the traffic light condition, one can easily say that mounted a camera on the vehicle can sense the which traffic light is on. However, according to [28], these types of sensors are only $90 \%$ accurate. Their team has developed another method for sensing traffic lights. They did this with the use of 3D mapping and a self-localization technique. Cameras were placed on the vehicle and gathered an image where the traffic light is likely to appear. The image of the traffic light is then fed to custom classifiers to determine the state. This method provides a $97 \%$ accuracy in determining the traffic light state.

The research of [29] also focused on the detection of obstacles in their work. Their method involves designing a hybrid encoder. They then used this encoder to analyze urban scenes. With the data, they have come up with different approaches to estimate obstacle locations.
In a similar study, the research of [30] focused on unmanned surface vehicles or USV. They used an advanced graphic model for semantic segmentation which incorporates the internal measurement unit and a stereo verification algorithm. The IMU readings are used to estimate the location of the vehicle. With this, equations were formulated and created an efficient algorithm for calibration detection. They then tested this with a USV with a mounted camera and found that it outperforms the conventional detection technologies.

\section{THEORETICAL CONSIDERATION}

The theoretical consideration for this study is the Systems Theory. Since assembling and constructing autonomous vehicles involve different control systems working together, Systems Theory is a great way to tackle the study theoretically.

\section{DATA AND RESULTS}

Figures 5,6 and 7 shows the block diagram in various scenarios

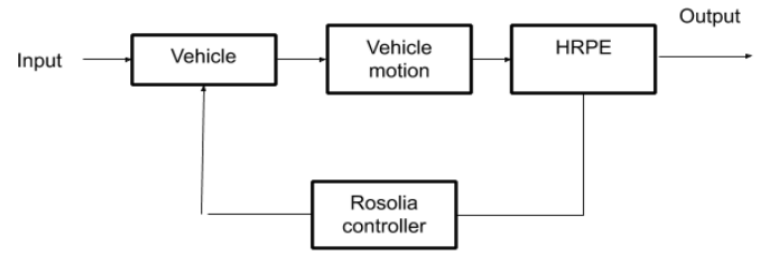

Figure 5: Block Diagram for undeveloped areas

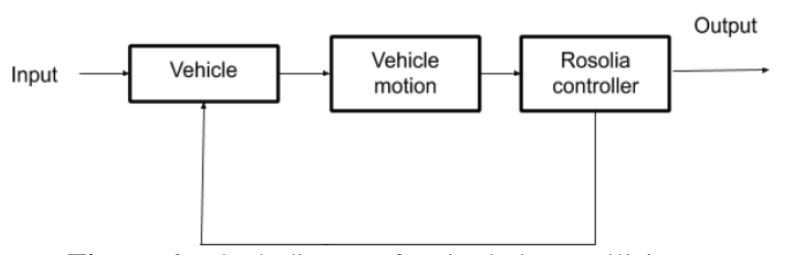

Figure 6: Block diagram for single lane collisions

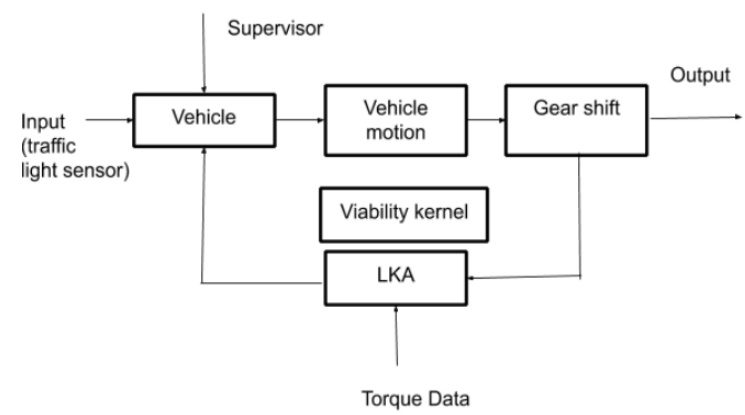

Figure 7: Block diagram for traffic lights and intersection

\section{ANALYSIS OF DATA}

Figure 5 represents the block diagram for when the automated vehicle is in an undeveloped area such as the slums or provinces with unfinished roads. The structures in this area are assumed to have poor features which will give the sensors difficulty in recognizing them. The input for this system will be those images obtained from the sensor. It is directly 
connected to the program of the automated vehicle. The process will be the vehicle's motion or its velocity on the road. After, it will undergo HRPE or the Heading reference assisted pose estimation developed by Wang and his team. This algorithm is mainly used for turning in undeveloped areas, but it can also be useful when going in a straight path as it is helpful for obstacle avoidance. This will then be fed back by the controller made by Rosolia's team. This controller includes barrier function which is mainly applied for collision avoidance. The controller will take over when a collision is likely to occur.

For Figure 6, the block diagram represents a single lane collision such as the collisions that happen in highways when a preceding vehicle has come to a stop immediately. This also applies to speed bumps, walking pedestrians, and stop signs if there are any. These applications are also inputting for the system. They were obtained from different camera sensors of the vehicle. Again, similar to the previous diagram, the process will be the vehicle's motion. This will then go to the controller of Rosolia's team. Since this is just a single lane collision, it only needs the barrier function produced by the controller.

The last block diagram (Figure 7) demonstrates the function of the automated vehicle when it is at an intersection with a traffic light. Its inputs are obtained from the camera sensors. Another input is also the supervisor algorithm as it detects future collisions in an intersection. This will then go to the vehicle's motion and then the gear shift schedule as proposed. This helps determine the proper amount of torque when slowing down or speeding up in case of a collision. If the vehicle is a semi-autonomous vehicle then it will feedback to the lane-keeping assist which has an external input of the steering wheel data of the human driver obtained from constant measurement. The block is useful in smoothing the transition of the control authority of the human driver and the autonomous driver or vice versa. This block can be added to the previous block diagrams, but it will be more difficult as there is no supervisor. If the vehicle is fully autonomous, then its feedback path will go directly to the viability kernels as to keep it on track.

\section{CONCLUSIONS}

The Philippines is one of the countries where driving can be a hazard. There are many unfinished roads, poor urban planning, heavy traffic, and more. With that, this paper focuses on the idea of autonomous vehicles being implemented in the Philippines. The paper will focus only on theoretical concepts gathered from different studies regarding the automated vehicle. No simulations were done. The students only focused on determining the advancement of the technologies of the A.V. They made block diagrams that contain different algorithms from different studies. The block diagrams are based on road scenarios that commonly happen to the Philippines. They include intersections with traffic lights, obstacle and collision avoidance in undeveloped areas such as the provinces and the slums, and single lane collisions such as speed bumps or when the preceding vehicle has come to an immediate stop. The algorithms used for those scenarios were the most applicable as to help guide the automated vehicle into a safer path, They include the barrier function which provides convenience in obstacle avoidance, the supervisor algorithm which is connected to a different autonomous vehicle and can control or guide them when a collision is predicted. Another helpful algorithm is the gear shift schedule where the vehicle determines the correct amount of torque necessary for the travel. It can help slow down or speed up when a collision is about to take place. With the data gathered and the diagrams constructed, the students concluded that the autonomous vehicle is not ready to be implemented in the Philippines. This is because there are no common algorithms for flooding, traffic enforcers, and more. Also, there are times where traffic lights are disabled which can be a problem for the vehicle.

\section{RECOMMENDATIONS}

This research solely involves the traffic congestion and road conditions of Metro Manila. It is highly recommended to research other parts of the country where traffic congestion is also evident i.e. Metro Cebu and Metro Davao. Currently, there are no fully automated vehicles in the market, since this research is about autonomous vehicles, it is recommended to research the application of improvements and innovations to adjust the settings of cars for traffic conditions in the Philippines. In other words, apply what this research has analyzed and concluded to cars, which are already available in the market, for their improvement and adjusting these cars for the traffic conditions in the Philippines.

This research also recommends research on further application of autonomous vehicles to help reduce the traffic congestion that the Philippines currently face. In research by [31], they conducted and designed traffic control systems, which can help reduce heavy traffic conditions and traffic congestions. Conducting research where coordinating traffic control systems and autonomous vehicles can greatly reduce traffic congestion and these control systems can have adaptive routing control to avoid furthermore traffic. Applying these conditions in the Philippines can greatly help reduce the evident traffic conditions.

Lastly, this research recommends for future researchers to research developing control systems embedded on autonomous vehicles which can determine what roads already have congestion. The research of [32] developed a paper where a control system that can evaluate, analyze, and monitor the traffic conditions via video data and TLCR (Traffic Lane Congestion Ratio). This traffic information can greatly help reduce congestion since it can detect what roads already have traffic. Conducting and applying this research on an autonomous vehicle wherein it can automatically determine what roads already have reduced traffic flow, can also help manage the traffic. 


\section{REFERENCES}

[1] J. R. F. Regidor, "Traffic congestion in metro Manila: Is the uvvrp still effective?'Philippine Engineering Journal. Vol. 34, No. 1, pp. 66-75,2013.

[2] D. W. Montgomery, "Public and Private Benefits of Autonomous Vehicles. Securing America's," Future Energy. 2018.

[3] E. R. P. Bondoc, F. P. M. Caparas, J.E.D.MacIas, V. T. Naculangga and J. E. Estrada, "An intelligent road traffic information system using text analysis in the most congested roads in Metro Manila," International Conference on Humanoid, Nanotechnology, Information Technology, Communication and Control, Environment and Management, HNICEM. 2019.

[4] M. Mydlarz and P. Skrzypczyński, "A Self-driving Car in the Classroom: Design of an Embedded, Behavior-Based Control System for a Car-Like Robot," Advances in Intelligent Systems and Computing. Vol. 920, pp. 367-378, 2020.

[5] A.Africa, D.Abaluna, and K. Pimentel, "Monitoring and control systems in agricultural machineries and equipment with a low-power smart antenna system," International Journal of Emerging Trends in Engineering Research. Vol. 8, No. 5. pp. 1860-1868, 2020.

[6] F. Mohdisharudden, H. Mohamed, Z. M. Rafaai, T. Y. W. Ho and M. S. Kamarudin, "Design and prototyping of a motorized legged robot with klann linkage mechanism," International Journal of Emerging Trends in Engineering Research. Vol.8, No. 5, pp. 1941-1945, 2020.

[7] D. Papageorgiou, T. Kastritsi and Z. Doulgeri, "A passive robot controller aiding human coaching for kinematic behavior modifications," Robotics and Computer-Integrated Manufacturing. Vol. 61,2020.

[8] G.E.C.Guevarra, A.R.T.Koizumi, J.N.B.Moreno, J.C.B.Reccion, C. M. O. Sy and J. R. B. Del Rosario, "Development of a quadrotor with vision-based target detection for autonomous landing," Journal of Telecommunication, Electronic and Computer Engineering. Vol. 10, Nos. 1-6, pp. 41-45, 2018.

[9] S. Alexandrova, M. Cakmak, K. Hsiao and L. Takayama, "Robot Programming by Demonstration with Interactive Action Visualizations," Robotics: Science and Systems. 2014.

[10] ABS-CBN news, "Metro Manila traffic congestion may be world's worst, says Waze," Retrieved from https://news.abs-cbn.com/business/10/29/19/metro-mani la-traffic-congestion-may-be-worlds-worst-says-waze. 20 19.

[11] Philkotse, "Are you sure you know these 5 major causes of traffic in the Philippines?" Retrieved from https://philkotse.com/safe-driving/top-5-major-causes-of -traffic-in-the-philippines.2019.

[12] C. C. Macadam, "Understanding and modeling the human driver," Vehicle System Dynamics. Vol. 40, Nos. 1-3, pp.101-134, 2003.

[13]M. Krampell, I. Solís-Marcos and M. Hjälmdahl, "Driving automation state-of-mind: Using training to instigate rapid mental model development," Applied Ergonomics. Vol. 83,2020.
[14]P. Bolia, T. Weiskircher, T and S. Müller, "Driver steering model for closed-loop steering function analysis," Vehicle System Dynamics. Vol. 52, No. 1, pp. 16-30, 2014.

[15] M. Hatakka, E. Keskinen, N. P. Gregersen, A. Glad and K. Hernetkoski, "From control of the vehicle to personal self-control; broadening the perspectives to driver education," Transportation Research Part F: Traffic Psychology and Behaviour. Vol. 5, No. 3, pp. 201-215, 2002.

[16]H. Ahn and D. Del Vecchio, "Safety Verification and Control for Collision Avoidance at Road Intersections," IEEE Transactions on Automatic Control. Vol. 63, No. 3, pp. 630-642, 2018.

[17] J. R. B. Del Rosario, A. A. Bandala and E. P. Dadios, "Multi-view multi-object tracking in an intelligent transportation system: A literature review," HNICEM 2017 - 9th International Conference on Humanoid, Nanotechnology, Information Technology, Communication and Control, Environment and Management. Vol.2018, pp. 1-4, 2017.

[18]H. Wang, R. Jiang, H. Zhang and S. Ge, "Heading Reference-Assisted Pose Estimation for Ground Vehicles," IEEE Transactions on Automation Science and Engineering. Vol. 16, No. 1, pp. 448-458, 2019.

[19] M. Forghani, J. McNew, D. Hoehener and D.Del Vecchio, "Design of driver-assist systems under probabilistic safety specifications near stop signs,"IEEE Transactions on Automation Science and Engineering. Vol. 13, No. 1, pp. 43-53, 2016.

[20]A. Liniger and J. Lygeros, "Real-Time Control for Autonomous Racing Based on Viability Theory,'IEEE Transactions on Control Systems Technology. Vol. 27, No. 2, pp. 464-478, 2019.

[21] C. R. He, W. B. Qin, N. Ozay and G. Orosz, "Optimal Gear Shift Schedule Design for Automated Vehicles: Hybrid System Based Analytical Approach," IEEE Transactions on Control Systems Technology. Vol. 26, No. 6, pp. 2078-2090, 2018.

[22] M. Pčolka, E. Žáčeková, S. Čelikovský and M. Šebek, "Toward a smart car: Hybrid nonlinear predictive controller with adaptive horizon,'IEEE Transactions on Control Systems Technology. Vol. 26, No. 6, pp. 1970-1981,2018.

[23] C. Sentouh, A. Nguyen, M. Benloucif and J. Popieul, "Driver-Automation Cooperation Oriented Approach for Shared Control of Lane Keeping Assist Systems,'IEEE Transactions on Control Systems Technology. 2018.

[24] Y. Chen, H. Peng and J. Grizzle, "Obstacle Avoidance for Low-Speed Autonomous Vehicles with Barrier Function," IEEE Transactions on Control Systems Technology. Vol. 26, No. 1, pp. 194-206,2018.

[25] U. Rosolia, S. De Bruyne and A. Alleyne, "Autonomous Vehicle Control: A Nonconvex Approach for Obstacle Avoidance," IEEE Transactions on Control Systems Technology. Vol. 25, No. 2, pp. 469-484, 2017.

[26] S. Lee and C. Chung, "Robust Multirate On-Road Vehicle Localization for Autonomous Highway Driving Vehicles," IEEE Transactions on Control Systems Technology. Vol. 25, No. 2, pp. 577-589, 2017. 
[27] K. Okamoto and P. Tsiotras, "Data-driven human driver lateral control models for developing haptic-shared control advanced driver assist systems," Robotics and Autonomous Systems. Vol. 114, pp. 155-171,2019.

[28] M. Hirabayashi, A. Sujiwo, A. Monrroy, S. Kato and M. Edahiro, "Traffic light recognition using high-definition map features," Robotics and Autonomous Systems. Vol. 111, pp. 62-72, 2019.

[29]A. Dairi, F. Harrou, M. Senouci and Y. Sun, "Unsupervised obstacle detection in driving environments using deep-learning-based stereovision," Robotics and Autonomous Systems. Vol. 100, pp. 287-301, 2018.

[30] B. Bovcon, R. Mandeljc, J. Perš and M. Kristan, "Stereo obstacle detection for unmanned surface vehicles by IMU-assisted semantic segmentation," Robotics and Autonomous Systems. Vol. 104, pp. 1-13, 2018.

[31] S. Lee, Y. Kim, H. Kahng, S. Lee, S. Chung, T. Cheong, K. Shin, J. Park and S. Kim, "Intelligent traffic control for autonomous vehicle systems based on machine learning," Expert Systems with Applications. Vol. 144, 2020.

[32] I. Stetsenko and O. Stelmakh, "Traffic lane congestion ratio evaluation by video data," Advances in Intelligent Systems and Computing. pp. 172-181, 2020. 